\title{
Invariant Correlational Entropy and Complexity of Quantum States
}

\author{
Valentin V. Sokolov ${ }^{1}$, B. Alex Brown ${ }^{2}$ and Vladimir Zelevinsky ${ }^{1,2}$ \\ ${ }^{1}$ Budker Institute of Nuclear Physics, 630090 Novosibirsk, Russia \\ ${ }^{2}$ Department of Physics and Astronomy and \\ National Superconducting Cyclotron Laboratory, \\ Michigan State University, East Lansing, MI 48824-1321, USA
}

\begin{abstract}
We define correlational (von Neumann) entropy for an individual quantum state of a system whose time-independent hamiltonian contains random parameters and is treated as a member of a statistical ensemble. This entropy is representation independent and can be calculated as a trace functional of the density matrix which describes the system in its interaction with the noise source. We analyze perturbation theory in order to show the evolution from the pure state to the mixed one. Exactly solvable examples illustrate the use of correlational entropy as a measure of the degree of complexity in comparison with other available suggestions such as basis-dependent information entropy. It is shown in particular that a harmonic oscillator in a uniform field of random strength comes to a quasithermal equilibrium; we discuss the relation between effective temperature and canonical equilibrium temperature. The notion of correlational entropy is applied to a realistic numerical caculation in the framework of the nuclear shell model. In this system, which reveals generic signatures of quantum chaos, correlational entropy and information entropy calculated in the mean field basis display similar qualitative behavior.
\end{abstract}




\section{INTRODUCTION}

The concept of entropy is fundamental for many branches of physics and other sciences dealing with systems which reveal a certain degree of complexity and disorder. As stressed in the monograph [1], "entropy is not a single concept but rather a family of notions". This monograph and the earlier review article [2] contain historical information and give many examples of different approaches to the idea of entropy and numerous applications.

In relation to quantum theory, the mainstream of development is formed by four main overlapping lines. They can be referred to as thermodynamical (Boltzmann - Gibbs) entropy, quantum ensemble (von Neumann) entropy, information (Shannon) entropy and dynamical (Kolmogorov - Sinai) entropy. Since the general description of a quantum system, including its interaction with the environment, time development and relaxation to equilibrium, can be given in terms of the density matrix [3, [4, the von Neumann definition seems to be the most fundamental. For a system in an equilibrium with a heat bath, the density matrix (and, accordingly, von Neumann entropy) is eqivalent to that in the canonical or grand canonical thermal ensemble. The evolution of a closed quantum many body (gas-like) system from a random initial state is shown [5,6] to lead to the same values of macroscopic observables as for the thermal equilibrium described by the microcanonical ensemble which has a clear semiclassical limit as the equipopulation on the energy surface in phase space. The ensemble entropy cannot be represented as an expectation value of a dynamic variable expressed by an operator in Hilbert space. However, being a trace functional of the density matrix, it is invariant under unitary basis transformations. For a pure wave function, as that of a stationary state in an isolated system, basis-independent von Neumann entropy vanishes. It reflects the mixed character of the quantum state with incomplete information.

Information entropy, with traditional applications in communication theory, is expressed in terms of probabilities rather than amplitudes. Therefore it is representation dependent being different for different choices of the set of mutually excluding events. In quantum systems, one can find information entropy of individual eigenstates with respect to a specific 
basis. All correlations between the amplitudes of different components of the wave function are suppressed in this definition. Averaging information entropy over some ensemble of quantum states, one gets a measure of average complexity of those states. At this stage, the similarity between information entropy and von Neumann ensemble entropy can emerge if one can establish an appropriate correspondence between the ensembles used in the two approaches and the basis utilized in calculating information entropy [7]. Thus, for canonical equilibrium thermal ensembles, the correlations are destroyed by the random interaction with the heat bath so that the density matrix is diagonal in the energy representation for the system. In this case, the eigenvalues of the density matrix give the occupancies of the stationary eigenstates of the isolated system which could be directly used for constructing information (= thermodynamical) entropy [3,8].

The so-called dynamical entropy, extensively studied during the last decade [9 14], is essentially information entropy applied to a random sequence of measurements of quantum observables. Apart from the intrinsic complexity of the system under study, this construction reflects special features of the quantum measurement process. This entropy depends not only on the initial state but, in addition, on the observable and on the way of performing the measurement. It can be defined so that it give classical Kolmogorov - Sinai entropy [15] in the corresponding limit of fine-grained phase space when the sequence of measurements may be described with the aid of symbolic dynamics 16 18, 14.

Information entropy used as a tool for quantifying the degree of complexity of individual quantum states [19 22] shows delocalization of the wave function in a given basis. However, as a rule one can find a basis, or a family of bases, which are singled out by physical arguments specific for each system. The delocalization length in such a representation manifests complex character of the state and can be quantitatively related to other signatures of quantum chaos. This exceptional role is naturally played by the coordinate representation in billiard-like cases [20] and by the quasienergy basis in the problems with a periodic perturbation [21]. For realistic many-body systems with strong interaction between constituents, the mean field represents the exceptional basis where the local correlations and fluctuations 
of adjacent stationary states are separated from their regular evolution along the spectrum [22].

As shown in large scale nuclear shell model calculations [23,7], the representation dependence of information entropy might be considered in some respects as an advantage which provides a useful physical measure of mutual relationship between the eigenbasis of the hamiltonian and the representation basis. Moreover, chaotic dynamics make different states with close excitation energy and the same values of exact constants of motion "look the same" [24], i.e. have similar observable properties. This is nothing but a microscopic picture of thermal equilibrium [25]. After averaging over a narrow energy window in a high level density region, information entropy in the mean field basis becomes a smooth function of excitation energy and carries [26,7] the same thermodynamic contents as thermal entropy found for the microcanonical distribution from the level density. Being calculated in a random basis, the magnitude of information entropy of generic states in a complex system is typically on the level predicted by random matrix theory and does not display any regular evolution along the spectrum.

The goal of the present paper is to explore the possibility of describing the degree of complexity of individual quantum states using the von Neumann definition of entropy and applying an external noise which converts a pure state into the mixed one. We do not consider the perturbation to be weak; therefore the resulting mixed state depends explicitly on the noise properties and gives a description of the ensemble "system plus noise". There exists a vast literature discussing the behavior of regular and chaotic systems under the change of parameters of the hamiltonian, see for example [27 37]. Multiple avoided crossings of the energy terms in a function of parameters reveal strong mixing and drive the system to the chaotic limit. The analogy of level dynamics with that of the one-dimensional gas of colliding particles is very productive for studying the spectral statistics [38,27,28, 34, 39, 40, Here we assume that the ensemble of the parameter values is defined by a distribution function and calculate the density matrix and von Neumann entropy for a given energy term. Using exactly solvable models, we show essential features of representation-independent entropy 
obtained according to this definition, its similarity to and distinction from information entropy. Even for the simplest systems as a harmonic oscillator in a random uniform field, the resulting steady states are far from trivial. We also give an example of a realistic numerical calculation for a many-body system of fermions (a nucleus ${ }^{24} \mathrm{Mg}$ ) which shows that our ensemble entropy ("correlational" entropy) is a smooth function of excitation energy and therefore may be used as a measure of the degree of complexity.

\section{DENSITY MATRIX AND CORRELATIONAL ENTROPY}

We consider a quantum system interacting with a surrounding. The interaction will be parameterized by a set of real parameters $\lambda$ in the hamiltonian, $H=H(\lambda)$. The energy spectrum of the system is assumed to be discrete. The eigenfunctions $|\alpha ; \lambda\rangle$ of the system, as well as its energy levels $E_{\alpha}(\lambda)$, evolve with $\lambda$. For a complicated system, the level crossings are avoided so one can continuously follow these energy terms.

At a fixed value of $\lambda$, one can use any complete orthonormal basis $|k\rangle$ to study the evolution of the eigenstates in terms of the amplitudes $C_{k}^{\alpha}(\lambda)$,

$$
|\alpha ; \lambda\rangle=\sum_{k} C_{k}^{\alpha}(\lambda)|k\rangle
$$

Instead of the wave function (1), one can also use the density matrix $\rho^{(\alpha)}$ whose elements are

$$
\rho_{k k^{\prime}}^{(\alpha)}(\lambda)=C_{k}^{\alpha}(\lambda) C_{k^{\prime}}^{\alpha *}(\lambda)
$$

$\rho^{(\alpha)}$ is a hermitian matrix in Hilbert space of the system. For a pure state $|\alpha ; \lambda\rangle$, the descriptions in terms of the wave function (11) and the density matrix (2) are fully equivalent. The obvious properties of the density matrix (2) are the normalization

$$
\operatorname{Tr} \rho^{(\alpha)}(\lambda)=1
$$

and the matrix identity 


$$
\left(\rho^{(\alpha)}(\lambda)\right)^{2}=\rho^{(\alpha)}(\lambda)
$$

which shows that the eigenvalues of this matrix can be only 0 or 1 . Actually, the density matrix (2) is diagonalized in the eigenbasis $\left|\alpha^{\prime} ; \lambda\right\rangle$. Only one eigenvalue, for the original state $\alpha^{\prime}=\alpha$, is equal to 1 while the rest of the eigenstates belong to the degenerate subspace with the zero eigenvalues of $\rho^{(\alpha)}(\lambda)$ so that $\rho^{(\alpha)}(\lambda)$ is the projection operator onto the state $|\alpha ; \lambda\rangle$. Inversely, these properties can be taken as a signature of a density matrix describing a pure state.

Now we assume that the interaction parameters $\lambda$ are random and have to be considered as members of an ensemble characterized by the normalized distribution function $\mathcal{P}(\lambda)$, $\int d \lambda \mathcal{P}(\lambda)=1$. Then the description in terms of a wave function becomes impossible, and our system is described by the density matrix (here and below the overline refers to ensemble averaged quantities)

$$
\rho_{k k^{\prime}}^{(\alpha)}=\overline{C_{k}^{\alpha} C_{k^{\prime}}^{\alpha *}}=\int d \lambda \mathcal{P}(\lambda) \rho_{k k^{\prime}}^{(\alpha)}(\lambda)
$$

This is still a hermitian matrix with trace equal to 1 . But, generally, the operator identity (4) is not valid anymore. The eigenvalues $\rho_{\nu}^{(\alpha)}$ of the matrix (5),

$$
\left.\left.\rho^{(\alpha)} \mid \nu\right)=\rho_{\nu}^{(\alpha)} \mid \nu\right)
$$

are nonnegative numbers between 0 and 1 ,

$$
\left(\rho_{\nu}^{(\alpha)}\right)^{2} \leq \rho_{\nu}^{(\alpha)}
$$

These eigenvalues can be interpreted as mean occupation numbers of the eigenstates $\mid \nu)$ for a system which was brought into the contact with an external source being originally in the intrinsic state $|\alpha\rangle$. The exceptional case of a pure wave function is recovered for a fixed parameter, $\mathcal{P}(\lambda)=\delta\left(\lambda-\lambda_{0}\right)$. In notations of eq. (白) and later on we distinguish the eigenstates $\mid \nu$ ) of the density matrix from the eigenstates $|\alpha\rangle$ of the hamiltonian by using the parentheses and the angular brackets, respectively; the dimensions of both sets are equal.

The statistical distribution of occupancies can be characterized by von Neumann entropy 


$$
S^{(\alpha)}=-\operatorname{Tr}\left\{\rho^{(\alpha)} \ln \rho^{(\alpha)}\right\}=-\sum_{\nu} \rho_{\nu}^{(\alpha)} \ln \rho_{\nu}^{(\alpha)}
$$

This entropy, being still attributed to a single original energy term $|\alpha\rangle$, reflects correlational properties of the system subject to different levels of noise. Therefore we will call it correlational entropy although the definition (8) is quite similar to that of standard thermodynamic entropy in canonical thermal ensembles [2]. In contrast to information entropy of a given complicated state in a fixed basis $|k\rangle$, which was used in the studies of quantum chaos $19,21,23$,

$$
I^{\alpha}=-\sum_{k} W_{k}^{\alpha} \ln W_{k}^{\alpha}, \quad W_{k}^{\alpha}=\left(C_{k}^{\alpha}\right)^{2}
$$

correlational entropy (8) is invariant and does not depend on the original basis $\{|k\rangle\}$ of simple configurations. Obviously, this is a consequence of correlations between different components of the eigenfunction which are absent in the probabilistic definition of eq. (9).

For a pure quantum state $|\alpha ; \lambda\rangle$, correlational entropy vanishes independently of the degree of complexity of the system. Thus, $S^{\alpha}$ characterizes the intrinsic term $|\alpha\rangle$ as a member of the statistical ensemble. In general, $S^{\alpha}$ has an order of magnitude of $\ln \tilde{N}_{\alpha}$ where $\tilde{N}_{\alpha}$ is a number of the eigenstates $\left.\mid \nu\right)$ which have the occupancies $\rho_{\nu}^{(\alpha)}$ noticeably different from zero. The maximum possible value of correlational entropy is $\ln N$ where $N$ is the dimension of Hilbert space. This value would correspond to the "microcanonical" density matrix, $\rho_{\nu}^{(\alpha)}=$ const $=1 / N$. Note that the information entropy [7] has the order of magnitude of $\ln N_{\alpha}$ where $N_{\alpha}$ is a number of essential components in the stationary wave function $|\alpha ; \lambda\rangle$ expressed in an original basis which was used in the definition of information entropy. Although formally the maximum value of information entropy is also $\ln N$, its typical value in the Gaussian orthogonal ensemble of random real symmetric hamiltonians stays, due to the fluctuations, on the level of $\ln (0.48 N)$, see [21,41,7]. In contrast to that, correlational entropy shows the degree of mixing, or decoherence, introduced by a given source of noise, regardless of the relationship between the resulting state and the original unperturbed basis. 
In next two sections we consider simple examples which allow us to obtain exact solutions and shed some light on main properties and physical meaning of new entropy.

\section{PERTURBATION THEORY}

We start with the case of a narrow noise range $\Delta \lambda$ that is small as compared to the scale of the parameter values which would lead to a considerable change of the wave functions. This is the perturbative regime. We can assume that the distribution function $\mathcal{P}(\lambda)$ is concentrated near $\lambda=0$ and find the perturbed wave function which starts its evolution for $\lambda \neq 0$ from the unperturbed state $|0\rangle$. The state $|0\rangle$ acquires the admixtures of states $|k \neq 0\rangle$ which are given by standard perturbation theory (we assume the absence of degeneracy).

With the hamiltonian $H=H_{0}+\lambda V$ where the perturbation $V$ is an off-diagonal operator in the eigenbasis of $H_{0}$, the density matrix (5) of the state $|0\rangle$ is, up to the second order in $\lambda$,

$$
\begin{gathered}
\rho_{l m}=\delta_{l 0} \delta_{m 0}\left(1-\overline{\lambda^{2}} \sum_{k \neq 0} \frac{\left|V_{k 0}\right|^{2}}{\epsilon_{k}^{2}}\right)-\bar{\lambda}\left(\delta_{l 0} \frac{V_{m 0}^{*}}{\epsilon_{m}}+\delta_{m 0} \frac{V_{l 0}}{\epsilon_{l}}\right) \\
+\overline{\lambda^{2}}\left\{\frac{V_{l 0} V_{m 0}^{*}}{\epsilon_{l} \epsilon_{m}}+\frac{\delta_{l 0}\left(1-\delta_{m 0}\right)}{\epsilon_{m}} \sum_{k \neq 0} \frac{V_{m k}^{*} V_{k 0}^{*}}{\epsilon_{k}}+\frac{\delta_{m 0}\left(1-\delta_{l 0}\right)}{\epsilon_{l}} \sum_{k \neq 0} \frac{V_{l k} V_{k 0}}{\epsilon_{k}}\right\} .
\end{gathered}
$$

Here $\bar{\lambda}$ and $\overline{\lambda^{2}}$ are the mean values over an ensemble of noise, and the notation $\epsilon_{k} \equiv E_{k}-E_{0}$ is used for the energy denominators. The density matrix (10) incorporates two effects, the redefinition of the original wave function $|0\rangle$ due to the perturbation $\lambda V$, and the transition from the pure state to the density matrix. The first effect is the only one if the perturbation is fixed while the second effect appears because of the ensemble distribution of perturbations.

It is easy to see that the first order correction to the density matrix does not change the purity condition (田), $\rho^{2}=\rho$. The decoherence occurs only in the second order. The diagonalization problem (6) for the matrix (10) can be solved to give, within a needed accuracy, two nonvanishing eigenvalues $\rho_{\nu}$ as the roots of the characteristic equation

$$
\rho(1-\rho)-w \overline{(\Delta \lambda)^{2}}=0
$$


and $N-2$ zero eigenvalues. As expected, the statistical mixing is driven by the mean square fluctuation $\overline{(\Delta \lambda)^{2}}=\overline{\lambda^{2}}-\bar{\lambda}^{2}$ of the noise level. The decoherence rate is determined by the joint action of all virtual transitions,

$$
w=\sum_{k}\left|v_{k}\right|^{2}, \quad v_{k} \equiv \frac{V_{k 0}}{\epsilon_{k}} .
$$

As seen from (11), only one state $\mid 0$ ) has the eigenvalue $\rho_{0}$ close to 1 ,

$$
\rho_{0}=1-w \overline{(\Delta \lambda)^{2}}
$$

The corresponding eigenfunction $\mid 0)=\sum_{k} \psi_{k}^{(0)}|k\rangle$ has a large component $\psi_{0}^{(0)} \approx 1-(w / 2) \bar{\lambda}^{2}$ of the unperturbed state $|0\rangle$ and small admixtures of other unperturbed states, $\psi_{k}^{(0)} \approx-\bar{\lambda} v_{k}$, $k \neq 0$; the corrections are of higher order if the distribution function $\mathcal{P}(\lambda)$ is even so that $\bar{\lambda}=0$.

In the approximation (11), the new state |1) appears with a small but nonzero occupation factor

$$
\rho_{1}=w \overline{(\Delta \lambda)^{2}}
$$

The eigenfunction of this state is localized mainly in the subspace orthogonal to the unperturbed state, $\psi_{k}^{(1)} \approx v_{k} w^{-1 / 2}, k \neq 0$. The presence of noise removes the isotropic degeneracy of this $(N-1)$-dimensional subspace by singling out the direction of the multidimensional vector of transition amplitudes $\left\{v_{k}\right\}$. Finally, the $N-2$ degenerate states with the zero occupation factors $\rho_{\nu}$ are orthogonal to the first two states. The high order perturbative corrections consequentially lift the remaining isotropy populating new combinations of original states. The decoherence process can be rather fast due to the added contributions of many distant admixtures so that perturbation theory can be valid at a very low noise level only. This "coherent decoherence" was discussed in a different context in 42. It is related to the selection of the most important (rainbow) diagrams in theory of disordered solids and in random matrix theory.

The perturbed occupancies lead to nonzero entropy (8) 


$$
S=-\rho_{0} \ln \rho_{0}-\rho_{1} \ln \rho_{1} \approx W(1-\ln W), \quad W=w \overline{(\Delta \lambda)^{2}} .
$$

The singularity at the origin implies the infinite slope, $d S / d W=-\ln W$, of growing entropy.

\section{TWO-LEVEL SYSTEMS}

\section{A. A two-level system with fluctuating level positions}

The simplest nontrivial but analytically solvable problem of a two-level system illustrates how correlational entropy is related to an interplay between the off-diagonal level mixing and their random diagonal displacements induced by the change of the parameter $\lambda$ within a range $\sim \Lambda$ defined by an ensemble $\mathcal{P}(\lambda)$.

Here we consider two interacting states with a fluctuating spacing so that the hamiltonian can be written, with the help of spin matrices, as

$$
H=\frac{1}{2}(\epsilon-\lambda) \sigma_{z}+V \sigma_{x} .
$$

The diagonalization of this hamiltonian at a fixed value of $\lambda$ is performed by the basis rotation through an angle $\varphi / 2$ defined by

$$
\sin \varphi=\frac{2 V}{\Delta(\lambda)}, \quad \cos \varphi=\frac{\epsilon-\lambda}{\Delta(\lambda)},
$$

where

$$
\Delta(\lambda)=\sqrt{(\epsilon-\lambda)^{2}+4 V^{2}}
$$

is the level spacing. The amplitudes of the upper, $(+)$, and of the lower, $(-)$, state can be written in the basis used in eq. (16) as

$$
C_{1}^{(+)}=\cos \frac{\varphi}{2}, \quad C_{2}^{(+)}=\sin \frac{\varphi}{2}, \quad C_{1}^{(-)}=-\sin \frac{\varphi}{2}, \quad C_{2}^{(-)}=\cos \frac{\varphi}{2} .
$$

The instantaneous density matrix (2) for each of the two states,

$$
\rho^{( \pm)}(\lambda)=\frac{1}{2}\left[1 \pm\left(\sigma_{x} \sin \varphi+\sigma_{z} \cos \varphi\right)\right]
$$


has the eigenvalues 1 and 0 , and the corresponding entropy vanishes. After averaging over the ensemble of the values of $\lambda$, we come to

$$
\rho^{( \pm)}=\frac{1}{2}\left[1 \pm\left(\sigma_{x} s+\sigma_{z} c\right)\right]
$$

where $c \equiv \overline{\cos \varphi}$ and $s \equiv \overline{\sin \varphi}$ are averaged quantities which in generally do not satisfy $s^{2}+c^{2}=1$. This matrix has two nonzero eigenvalues,

$$
\rho_{\nu}=\frac{1}{2}(1+\nu r), \quad r=\sqrt{s^{2}+c^{2}}, \quad \nu= \pm 1
$$

which are the same for both original states $( \pm)$. Therefore both states possess the same correlational entropy

$$
S=-\frac{1}{2}\left[(1+r) \ln \frac{1+r}{2}+(1-r) \ln \frac{1-r}{2}\right] .
$$

The exact result depends on the ensemble but, as a function of the averaging range $\Lambda$, the entropy value (23) evolves from $S=0$ at $\Lambda=0$ to $S=\ln 2$ for the equipartition of the occupancies at large $\Lambda$ when $\rho_{\nu}=1 / 2$.

As an example, we consider the behavior of correlational entropy for the ensemble with the uniform distribution of $\lambda$ in the interval $(-\Lambda, \Lambda)$. With the definition (18) we have in this case

$$
s=\frac{V}{\Lambda} \ln \frac{\epsilon+\Lambda+\Delta(-\Lambda)}{\epsilon-\Lambda+\Delta(\Lambda)}, \quad c=\frac{1}{2 \Lambda}[\Delta(-\Lambda)-\Delta(\Lambda)]
$$

The simplest case corresponds to the original degeneracy, $\epsilon=0$, when

$$
s=\frac{\tau}{2} \ln \frac{\sqrt{1+\tau^{2}}+1}{\sqrt{1+\tau^{2}}-1}, \quad c=0, \quad \tau=\frac{2 V}{\Lambda} .
$$

The averaged parameter $s$ is small, $s \approx \tau \ln (2 / \tau)$, if the noise level is high and the intrinsic mixing is weak compared to the typical random level spacing, $\tau \ll 1$. At strong mixing, $\tau \gg 1, s$ reaches 1 . In this limit the levels are split by the dynamic interaction and the noise is ineffective in changing the population and reaching the decoherence. The entropy value correspondingly evolves from $\ln 2$ to 0 . This behavior is opposite to the evolution of information entropy of the same states, 


$$
I=-\frac{1+\cos \varphi}{2} \ln \frac{1+\cos \varphi}{2}-\frac{1-\cos \varphi}{2} \ln \frac{1-\cos \varphi}{2}
$$

as a function of the mixing strength for a fixed diagonal spacing; $I \rightarrow 0$ at weak mixing and $I \rightarrow \ln 2$ at strong mixing. In this example, information entropy measures merely the delocalization of the eigenstates with respect to the original basis.

In Fig. 1 we show correlational entropy (23) as a function of $\tau$ for different values of the ratio $\chi=\epsilon / \Lambda$. At $\chi<1$ (Figs. 1 a and $1 b$ correspond to $\chi=0.2$ and 0.6 , respectively), the results are qualitatively similar to those at $\epsilon=0$, eqs. (23) and (25). With no dynamic mixing, $\tau=0$, entropy is close to its maximum value, $S \approx \ln 2-\chi^{2}$ at small $\chi$. As the relative strength $\tau$ of the dynamic mixing increases, the value of entropy drops, $S \approx[1+\ln (4 \tau)] /(4 \tau)$ at large $\tau$. At $\chi \geq 1$, the entropy behavior changes since the noise level is not sufficient for covering the original level spacing, see Figs. $1 c$ and $1 d$, for $\chi=1$ and 5 , respectively. In this situation, weak dynamic mixing increases entropy which starts from zero at $\tau=0$ and reaches its maximum value, much lower than $\ln 2$, at $\tau=\chi$. With the mixing strength increasing further, the level repulsion prevails which leads again to very low entropy.

A complementary picture of Fig. 2 shows correlational entropy (23) as a function of the relative noise strength $\chi^{-1}=\Lambda / \epsilon$ for four different values of the intrinsic dynamical mixing $\zeta=2 V / \epsilon=\tau / \chi$. Generally, entropy increases with $\chi^{-1}$ and asymptotically approaches the maximum value $\ln 2$. For weak dynamical mixing $\zeta$, the growth of correlational entropy is very steep in the vicinity of the point $\chi=1$ where the accidental level crossing is possible for the first time, compare the change of behavior at this point in Fig. 1. The stronger is the dynamical mixing the smoother gets the increase of entropy. Entropy remains low as long as the intrinsic level repulsion governed by the parameter $\zeta$ dominates.

\section{B. A two-level system with fluctuating mixing}

We consider the same hamiltonian (16) assuming now that the diagonal elements are fixed (we change the notation $(\epsilon-\lambda) \rightarrow \epsilon$ ) but the off-diagonal coupling $V$ fluctuates. For definiteness, we assume that $V$ is uniformly distributed between $v>0$ and $-v$. This 
situation is simpler than that considered in the previous subsection because the only relevant parameter is that of perturbation theory, the ratio

$$
\kappa=\frac{2 v}{\epsilon}
$$

of the level spacing due to the mixing to the unperturbed spacing.

The eigenvalues of the density matrix are still given by eq. (22). Due to symmetry of perturbation, the average value of $\sin \varphi$ vanishes while the average value of $\cos \varphi$ is

$$
c=\frac{1}{\kappa} \ln \left(\kappa+\sqrt{1+\kappa^{2}}\right)
$$

With weak mixing, $c \approx 1-\left(\kappa^{2} / 6\right)$, and entropy (23) is low,

$$
S \approx\left(\kappa^{2} / 12\right) \ln \left(\kappa^{2} / 12\right), \quad \kappa \ll 1
$$

When the mixing intensity increases, $c$ falls down, and entropy approaches its limiting value for equipopulated levels,

$$
S \approx \ln 2-\frac{c^{2}}{2} \approx \ln 2-\frac{1}{2}\left(\frac{\ln 2 \kappa}{\kappa}\right)^{2} . \quad \kappa \gg 1 .
$$

One can note that here the off-diagonal mixing plays a "natural" role of noise which creates entropy, in contrast to the previous example when it has been stabilizing the system by generating dynamic repulsion as a counterpoise to random noise; that situation can be considered as a prototype of the localization in disordered solids when the levels in the wells connected by tunneling fluctuate.

\section{Spin in a random field}

This two-state model is more general in the sense that both the mixing strength and the level spacing are fluctuating here. On the other hand, the level spacing in this model does not depend on noise so there is no level crossings. The hamiltonian describes a spin $1 / 2$ aligning along a random field, 


$$
H=\vec{\sigma} \cdot \mathbf{n},
$$

where the unit vector $\mathbf{n}$ has a random direction $(\theta, \varphi)$. Information entropy (9) of the eigenstates for a fixed field orientation,

$$
I^{+}=I^{-}=-\frac{1}{2}\left\{\ln \frac{1-\cos ^{2} \theta}{4}+|\cos \theta| \ln \frac{1+|\cos \theta|}{1-|\cos \theta|}\right\},
$$

does not depend on the azimuthal angle $\varphi$. The density matrices (2) of the eigenstates $| \pm\rangle$ with the eigenenergies \pm 1 can be written as

$$
\rho^{( \pm)}(\mathbf{n})=\frac{1}{2}(1 \pm \vec{\sigma} \cdot \mathbf{n})
$$

and depend, contrary to (32), on both coordinate angles.

After the averaging over the ensemble of angles, we find the eigenvalues $\rho_{\nu}$ of the density matrix (间)

$$
\rho_{\nu}=\frac{1}{2}(1+\nu r), \quad \nu= \pm 1
$$

for any of the two original states $( \pm$ ). Here (compare eq. (22))

$$
r \equiv \sqrt{\overline{\mathbf{n}}^{2}}=\sqrt{c^{2}+s_{+} s_{-}}, \quad c \equiv \overline{\cos \theta}, \quad s_{ \pm} \equiv \overline{\sin \theta \exp ( \pm i \varphi)} ; \quad 0 \leq r \leq 1 .
$$

This allows one to find correlational entropy in the form (23),

$$
S=-\frac{1}{2}\left\{\ln \frac{1-r^{2}}{4}+r \ln \frac{1+r}{1-r}\right\} \text {. }
$$

The expressions (32) and (36) have identical structure. Correlational entropy reduces to information one in the case when the field direction is uniformly distributed on the surface of a cone with the fixed polar angle $\theta$. The average density matrix is then diagonal in the original $z$-representation which is singled out by asimuthal symmetry of the noise distribution. The two entropies coincide numerically at a point $\theta=\theta_{0}$ where the effective angle $\theta_{0}$ is defined by the condition $\cos \theta_{0}=r$. 


\section{Random matrix ensembles}

The $2 \times 2$ Hamiltonian matrix of an arbitrary two-level system can be presented as

$$
H=\frac{1}{2}(u+\vec{\sigma} \cdot \mathbf{v})
$$

in terms of one scalar, $u=\operatorname{Tr} H$, and one vector, $\mathbf{v}=v \mathbf{n}$, parameters. The vector $\mathbf{v}$ is restricted to the $(x, z)$ plane if time reversal invariance holds and becomes three-dimensional otherwise. Its magnitude $v$ gives the level spacing. Both entropies $I$ and $S$ depend only on the unit vector $\mathbf{n}$ so that formulae (32-36) of the previous subsection remain valid independently of statistical properties of $u$ and $v$.

If all parameters of a hamiltonian matrix or some part of them can be random, the hamiltonian belongs to a random matrix ensemble. Gaussian orthogonal, GOE, and Gaussian unitary, GUE, ensembles of large random matrices attract special attention for modeling chaotic dynamics in quantum systems. In Gaussian ensembles, the hamiltonian matrix elements calculated in a fixed basis are taken as mutually independent Gaussian random variables. The idea of complete chaos implies that their distribution be invariant with respect to the choice of the representation basis $|k\rangle$. This invariance provides a rigid connection between variances of all matrix elements. However, it has been discovered that one gains additional possibility of describing special effects such as dynamical localization by permitting more flexible conditions for the variances. Some preferential basis there exists then where the localization takes place. This basis should be defined in each case in accordance with the concrete physical situation. Basis dependent information entropy or/and inverse participation ratio are used to describe the localization, see for example studies of the banded random matrix ensemble [43,44]. Correlational entropy seems to present a universal and invariant characteristic of chaotic dynamics in presence of localization. We hope to return to this problem elsewhere.

In Gaussian random matrix ensembles, all eigenstates have the same statistical distributions so that any of them can represent the generic features. Here we will only point 
out the simplest properties of correlational entropy using the example of two-level Gaussian ensembles. According to (35-36), this entropy reaches its maximum value of $\ln 2$ under the condition of $\overline{\mathbf{n}}=0$. This is obviously the case for invariant (orthogonal or unitary) ensembles where the vector $\mathbf{n}$ is distributed isotropically. For both the ensembles, mean information entropy,

$$
\overline{I_{G O E}}=\frac{1}{2 \pi} \int_{0}^{2 \pi} d \varphi I(\varphi)=2 \ln 2-1
$$

or

$$
\overline{I_{G U E}}=\frac{1}{4 \pi} \int d \Omega I(\theta)=\frac{1}{2}
$$

is always lower than $S$. In eqs. (38) and (39) the angular coordinates of the unit vector $\mathbf{n}$ are used, $0 \leq \varphi \leq 2 \pi$ and $0 \leq \theta \leq \pi$. The instantaneous quantity $I(\mathbf{n})$ passes the maximum value of $\ln 2$ at the certain orientations of the vector $\mathbf{n}$, as at the point $\theta=\pi / 2$ in eq. (32), remaining smaller everywhere else. In fact, the relation $\bar{I}<S$ is valid for the Gaussian ensembles of an arbitrary dimension $N$. As we have already mentioned, for $N \gg 1$, correlational entropy is equal to $S=\ln N$ whereas mean information entropy reaches in this limit the value $\bar{I}=\ln (0.48 N)$.

At the same time, it can be shown by direct calculation that correlational entropy remains at the maximum value also when variances of all matrix elements have arbitrary values and the vector $\mathbf{n}$ ceases to be fully isotropic. Indeed, the probability distributions for ensembles of real symmetric matrices,

$$
\mathcal{P}_{\text {sym }}(\mathbf{n}) d \varphi=\frac{\sigma \varsigma}{\varsigma^{2} \cos ^{2} \varphi+\sigma^{2} \sin ^{2} \varphi} \frac{d \varphi}{2 \pi}
$$

and hermitian matrices,

$$
\mathcal{P}_{h e r m}(\mathbf{n}) d \Omega=\frac{\sigma^{2} \varsigma_{1}^{2} \varsigma_{2}^{2}}{\left[\varsigma_{1}^{2} \varsigma_{2}^{2} \cos ^{2} \theta+\sigma^{2}\left(\varsigma_{1}^{2} \sin ^{2} \varphi+\varsigma_{2}^{2} \cos ^{2} \varphi\right) \sin ^{2} \theta\right]^{3 / 2}} \frac{d \Omega}{4 \pi},
$$

remain even which yields $\overline{\mathbf{n}}=0$ as before. In eq. (41), $\sigma^{2}=\left(\sigma_{1}^{2}+\sigma_{2}^{2}\right) / 2$ whereas $\sigma_{1,2}$ and $\varsigma_{1,2}$ are variances of diagonal and off-diagonal matrix elements, respectively. 
Correlational entropy drops to zero only when the off-diagonal matrix element vanishes identically. The randomness of interaction is of major importance. On the contrary, information entropy is sensitive to the ratios of variances which depend on the representation basis.

\section{HARMONIC OSCILLATOR IN A RANDOM FIELD}

Here we consider a problem with infinite Hilbert space, namely that of a linear harmonic oscillator in a uniform external field of a random strength. The diagonalization of the hamiltonian at any fixed parameter value is elementary but the diagonalization of the density

matrix and calculation of correlational entropy are not trivial and we did not encounter the full solution in the literature although our results slightly overlap with those of ref. 45.

\section{A. The model}

At a given strength $F$, the hamiltonian of the problem,

$$
H \equiv H_{0}-F x=\frac{1}{2 m} p^{2}+\frac{1}{2} m \omega^{2}\left(x-\frac{F}{m \omega^{2}}\right)^{2}-\frac{F^{2}}{2 m \omega^{2}},
$$

describes the harmonic oscillator with the shifted equilibrium point, unchanged frequency and the energy spectrum

$$
E_{n}(\lambda)=\hbar \omega\left(n+1 / 2-\lambda^{2}\right)
$$

where the integer $n$ plays the role of the exact quantum numbers $\alpha$ in (11), and the dimensionless "noise" parameter

$$
\lambda=\frac{F}{\left(2 \hbar m \omega^{3}\right)^{1 / 2}}
$$

displaces the spectrum as a whole (no crossing in this model).

The "natural" basis $|k\rangle$ of equidistant non-shifted states of a noiseless system, $\lambda=0$, is built with the annihilation and creation operators, 


$$
x=\frac{x_{0}}{\sqrt{2}}\left(a^{\dagger}+a\right), \quad p=\frac{i \hbar}{\sqrt{2} x_{0}}\left(a^{\dagger}-a\right), \quad x_{0}=\sqrt{\frac{\hbar}{m \omega}} .
$$

The shift of the equilibrium along the $x$-axis to the point $\bar{x}=F / m \omega^{2}=\sqrt{2} x_{0} \lambda$ is the unitary transformation

$$
D(\lambda)=e^{-i \sqrt{2} \lambda x_{0} p / \hbar}=e^{\lambda\left(a^{\dagger}-a\right)}=D^{\dagger}(-\lambda)
$$

with the obvious properties

$$
D(\lambda) a D(-\lambda)=a-\lambda, \quad D(\lambda) a^{\dagger} D(-\lambda)=a^{\dagger}-\lambda
$$

so that (compare $(43))$

$$
H(\lambda)=D(\lambda) H_{0} D(-\lambda)-\lambda^{2} \hbar \omega
$$

The eigenvectors $|n ; \lambda\rangle$ at a given $\lambda$ are obtained from the original non-shifted states $|n\rangle \equiv$ $|n ; \lambda=0\rangle$ by the shift operator $D(\lambda)$,

$$
|n ; \lambda\rangle=D(\lambda)|n\rangle
$$

Therefore the amplitudes of the eigenvectors (1) with respect to the noiseless basis $|k\rangle$ are given by the matrix elements

$$
C_{k}^{n}(\lambda)=\langle k|D(\lambda)| n\rangle
$$

of the shift operator (46). The instantaneous eigenvector $|n ; \lambda\rangle$ carries an average number of the original quanta equal to

$$
\bar{n}=\left\langle n ; \lambda\left|a^{\dagger} a\right| n ; \lambda\right\rangle=n+\lambda^{2}
$$

\section{B. Integral equation for the density matrix}

For the noise ensemble characterized by the (positively defined) distribution function $\mathcal{P}(\lambda)$, the density matrix (5) of the energy term $|n\rangle$ is 


$$
\rho_{k k^{\prime}}^{(n)}=\int d \lambda \mathcal{P}(\lambda)\langle k|D(\lambda)| n\rangle\left\langle n\left|D^{\dagger}(\lambda)\right| k^{\prime}\right\rangle
$$

where the unitarity of the $D$-transformation was used. This matrix represents the density operator

$$
\rho^{(n)}=\int d \lambda \mathcal{P}(\lambda) D(\lambda)|n\rangle\langle n| D^{\dagger}(\lambda)
$$

in the noiseless basis. It is convenient to project instead the eigenvalue problem

$$
\left.\left.\rho^{(n)} \mid \nu\right)=\rho_{\nu}^{(n)} \mid \nu\right)
$$

onto the set of states $|n ; \lambda\rangle$ with the noise $\lambda$ treated as the representation variable. Generally speaking, this set of states is not complete and maps the original problem on the subspace of eigenstates which belong to all nonvanishing eigenvalues $\rho_{\nu}^{(n)}$. In the new representation, the eigenfunctions

$$
\phi_{\nu}^{(n)}(\lambda)=A_{\nu}^{(n)} \sqrt{\mathcal{P}(\lambda)}\left\langle n\left|D^{\dagger}(\lambda)\right| \nu\right)
$$

where $A_{\nu}^{(n)}$ are normalization constants, satisfy the integral equation

$$
\int d \lambda^{\prime} \rho^{(n)}\left(\lambda, \lambda^{\prime}\right) \phi_{\nu}^{(n)}\left(\lambda^{\prime}\right)=\rho_{\nu}^{(n)} \phi_{\nu}^{(n)}(\lambda)
$$

with the real symmetric kernel

$$
\rho^{(n)}\left(\lambda, \lambda^{\prime}\right)=\sqrt{\mathcal{P}(\lambda)}\left\langle n\left|D\left(\lambda^{\prime}-\lambda\right)\right| n\right\rangle \sqrt{\mathcal{P}\left(\lambda^{\prime}\right)}
$$

The diagonal elements of this kernel are simply the probabilities of the noise distribution,

$$
\rho^{(n)}(\lambda, \lambda)=\mathcal{P}(\lambda)
$$

They describe the ensemble itself and do not depend on the term $n$ under consideration.

The kernel (57) can be expressed as

$$
\rho^{(n)}\left(\lambda, \lambda^{\prime}\right)=\sqrt{\mathcal{P}(\lambda) \mathcal{P}\left(\lambda^{\prime}\right)} e^{-\left(\lambda-\lambda^{\prime}\right)^{2} / 2} L_{n}\left[\left(\lambda-\lambda^{\prime}\right)^{2}\right] .
$$

in terms of the Laguerre polynomial defined by the series 


$$
L_{n}(\xi)=\sum_{m=0}^{n} \frac{n !}{(m !)^{2}(n-m) !} \xi^{2 m} .
$$

It follows from (53,55) that the orthonormalized, $\left(n ; \nu^{\prime} \mid n ; \nu\right)=\delta_{\nu^{\prime} \nu}$, subset of eigenvectors belonging to nonzero eigenvalues $\rho_{\nu}^{(n)}$ is expressed in terms of the functions (55) as

$$
\mid n ; \nu)=\left(\rho_{\nu}^{(n)}\right)^{-1 / 2} \int d \lambda \sqrt{\mathcal{P}(\lambda)} \phi_{\nu}^{(n)}(\lambda)|n ; \lambda\rangle
$$

if the mutually orthogonal solutions (55) of the integral equation (56) with the hermitian kernel (57) are normalized to unity which corresponds to the choice of $A_{\nu}^{(n)}=\left(\rho_{\nu}^{(n)}\right)^{-1 / 2}$.

Starting with the completeness condition $\left.\sum_{\nu} \mid n ; \nu\right)(n ; \nu \mid=1$, one easily comes to the kernel representation

$$
\rho^{(n)}\left(\lambda, \lambda^{\prime}\right)=\sum_{\nu} \rho_{\nu}^{(n)} \phi_{\nu}^{(n)}(\lambda) \phi_{\nu}^{(n)}\left(\lambda^{\prime}\right)
$$

In the original oscillator basis $|k\rangle \equiv|k ; 0\rangle$, the components of the eigenvectors (55) are

$$
\left.\left(\psi_{\nu}^{(n)}\right)_{k}=\langle k| n ; \nu\right)=\left(\rho_{\nu}^{(n)}\right)^{-1 / 2} \int d \lambda \sqrt{\mathcal{P}(\lambda)} \phi_{\nu}^{(n)}(\lambda)\langle k|D(\lambda)| n\rangle .
$$

Matrix elements of the shift operator are equal to

$$
\langle k|D(\lambda)| n\rangle=e^{-\lambda^{2} / 2} \sqrt{\frac{n !}{k !}}(-\lambda)^{k-n} L_{n}^{k-n}\left(\lambda^{2}\right)
$$

where

$$
L_{n}^{q}(\xi)=\sum_{m=0}^{n} \frac{(n+q) !}{m !(m+q) !(n-m) !} \xi^{2 m}
$$

is the associated Laguerre polynomial, $L_{n}^{0}(\xi) \equiv L_{n}(\xi)$. Due to the symmetry property

$$
L_{n}^{k-n}(\xi)=\frac{k !}{n !}(-\xi)^{n-k} L_{k}^{n-k}(\xi)
$$

the matrix (64) is symmetric with respect to $k$ and $n$.

Up to this point, the results are valid for any energy term $|n ; \lambda\rangle$ and an arbitrary distribution function $\mathcal{P}(\lambda)$. 


\section{Gaussian noise}

As an example of practical importance, we show here the exact solution for an oscillator originally in the ground state, $n=0$, interacting with a source of Gaussian noise of a certain width $\Lambda$. The distribution function of the noise intensity is in this case

$$
\mathcal{P}(\lambda)=\frac{1}{\sqrt{2 \pi \Lambda^{2}}} e^{-\lambda^{2} / 2 \Lambda^{2}},
$$

and the ground state density matrix (59) reads in the $\lambda$-representation (we omit the superscript $n=0)$

$$
\rho\left(\lambda, \lambda^{\prime}\right)=\frac{1}{\sqrt{2 \pi \Lambda^{2}}} e^{-\left(\lambda^{2}+\lambda^{\prime 2}\right) / 4 \Lambda^{2}} e^{-\left(\lambda-\lambda^{\prime}\right)^{2} / 2},
$$

Using the properties of the Hermite polynomials $H_{\nu}(x)$, one can represent the kernel (68) as

$$
\rho\left(\lambda, \lambda^{\prime}\right)=\sum_{\nu=0}^{\infty} \rho_{\nu} \phi_{\nu}(\lambda) \phi_{\nu}\left(\lambda^{\prime}\right)
$$

in terms of the set of orthonormalized functions

$$
\phi_{\nu}(\lambda)=\left(\frac{\sinh \eta}{\pi}\right)^{\frac{1}{4}} \frac{1}{\sqrt{2^{\nu} \nu !}} e^{-\lambda^{2} \sinh \eta / 2} H_{\nu}(\sqrt{\sinh \eta} \lambda),
$$

where

$$
\rho_{\nu}=2 \sinh (\eta / 2) e^{-(\nu+1 / 2)},
$$

and the the parameter $\eta, 0 \leq \eta<\infty$, is defined by

$$
\sinh \eta=\frac{\sqrt{1+4 \Lambda^{2}}}{2 \Lambda^{2}} .
$$

The representation (69) obviously solves the eigenvalue problem (56) for the ground state term. The normalization of the density matrix $\sum_{\nu=0}^{\infty} \rho_{\nu}=1$ can be checked directly. The value of correlational entropy defined by this density matrix is

$$
S=-\sum_{\nu} \rho_{\nu} \ln \rho_{\nu}=\frac{\eta}{2} \operatorname{coth}(\eta / 2)-\ln [2 \sinh (\eta / 2)] .
$$


The distribution function for the number of original quanta is given by the diagonal matrix elements of the density matrix in the unperturbed basis $|k\rangle$,

$$
f_{k}=\langle k|\rho| k\rangle=\int d \lambda \mathcal{P}(\lambda)|\langle k \mid 0 ; \lambda\rangle|^{2}=\sqrt{\frac{2}{\pi}} \frac{\Gamma(k+1 / 2)}{\Gamma(k+1)} \frac{\sinh (\eta / 2)}{(\cosh \eta)^{k+1 / 2}}
$$

The average number of excited quanta equals

$$
\bar{k}=\sum_{k=0}^{\infty} k f_{k}=\int d \lambda \lambda^{2} \mathcal{P}(\lambda)=\overline{\lambda^{2}}=\Lambda^{2}
$$

which leads to the simple value of average energy of the term

$$
\bar{E}=\operatorname{Tr}(\rho H)=\hbar \omega(\bar{k}+1 / 2)-\overline{F x}=\hbar \omega\left(1 / 2-\Lambda^{2}\right),
$$

in agreement with (43) and (51) for $n=0$. The average energy of a given term is always lowered by the presence of noise; however, this energy cannot be attributed to the oscillator itself because it includes the contribution of the external noise source.

According to $(\sqrt{75})$ and $(\sqrt{72})$, the mean number of original quanta excited by the external field can be written as

$$
\bar{k}=\frac{1}{4 \sinh ^{2}(\eta / 2)}
$$

Introducing $\bar{k}$ as a new parameter,

$$
\sinh \frac{\eta}{2}=1 /(2 \sqrt{\bar{k}}), \quad \cosh \eta=1+1 /(2 \bar{k})
$$

we obtain the distribution of quanta (74) in the form

$$
f_{k}=(2 \bar{k})^{-1 / 2} \frac{(2 k) !}{2^{2 k}(k !)^{2}} \frac{1}{[1+1 /(2 \bar{k})]^{k+1 / 2}}
$$

When the external perturbation is turned off, $\bar{k} \rightarrow 0$ and $f_{k} \rightarrow \delta_{k 0}$. In the opposite limit of strong noise, the mean number of excited quanta is large, $\bar{k} \gg 1$, and eq. (79) reduces to the chi-square (Porter-Thomas) distribution,

$$
f_{k}=(2 \pi \bar{k} k)^{-1 / 2} \exp [-k /(2 \bar{k})], \quad \bar{k} \gg 1
$$


We have to mention that, in the case of a fixed perturbation of a strength $\lambda$, we would have a pure coherent state of a shifted oscillator with the Poisson distribution

$$
f_{k}=e^{-\bar{k}} \frac{\bar{k}^{k}}{k !}
$$

which reduces to the Gaussian distribution near the center $k=\bar{k}$ in the classical limit of $\bar{k} \gg 1$. The width of the coherent state in the occupation number representation is $(\Delta k)^{2}=\bar{k}$ and therefore the relative uncertainty of this number is small, $\Delta k / \bar{k}=\bar{k}^{-1 / 2}$. Contrary to that, in our random noise ensemble (80) the state is mixed and the fluctuations of the number of quanta are not suppressed even in the classsical limit,

$$
\overline{(\Delta k)^{2}}=2 \bar{k}^{2}
$$

The situation is similar to that in the boson intensity interferometry (Hanbury Brown Twiss) for many incoherent sources, see for example [46.

\section{Relation to thermodynamics}

The eigenfunctions (70) of the density matrix (68) in the $\lambda$-representation formally coincide with the coordinate wave functions $(\xi \mid \nu)$ for a linear oscillator with the dimensionless "coordinate" $\xi \equiv \sqrt{\sinh \eta} \lambda$. The dimensionless hamiltonian for such an oscillator would be

$$
\mathcal{H}=\frac{1}{2}\left(\xi^{2}-d^{2} / d \xi^{2}\right)=\left(\alpha^{\dagger} \alpha+1 / 2\right)
$$

where the annihilation and creation operators

$$
\alpha=\frac{1}{\sqrt{2}}(\xi+d / d \xi), \quad \alpha^{\dagger}=\frac{1}{\sqrt{2}}(\xi-d / d \xi)
$$

are introduced. The energy spectrum of the operator (83), $\varepsilon_{\nu}=(\nu+1 / 2)$, is equidistant and quantized in $\lambda$-independent units at any noise magnitude. Since the density operator is diagonal in the basis of the eigenfunctions of (83), it is tempting to interpret our results as an equilibrium reached by the system under the influence of the noise. The equilibrated ("dressed") system is represented by the effective oscillator (83). We can assign the physical 
frequency $\omega$ to this oscillator and interpret the corresponding occupation number spectrum (71) as the Planck formula with the effective temperature

$$
T=\frac{\hbar \omega}{\eta}
$$

Under this identification, the temperature scale is related to the range of noise,

$$
\Lambda=\frac{1}{2 \sinh (\hbar \omega / 2 T)}
$$

or to the mean square value of the random force,

$$
\left(\overline{F^{2}}\right)^{1 / 2} x_{0}=\frac{1}{\sqrt{2}} \frac{\hbar \omega}{\sinh (\hbar \omega / 2 T)}
$$

The density operator can be represented in terms of the effective oscillator defined above as

$$
\left.\rho=\exp (-\hbar \omega \mathcal{H} / T)=\sum_{\nu=0}^{\infty} \rho_{\nu} \mid \nu\right)\left(\nu \mid, \quad \rho_{\nu}=2 \sinh \left(\frac{\hbar \omega}{2 T}\right) \exp \left(-\frac{\hbar \omega}{T}(\nu+1 / 2)\right) .\right.
$$

The effective oscillator is in the conventional thermodynamic equilibrium with a heat bath of temperature $T$, eq. (85). In an ordinary way, we can define its free energy

$$
\mathcal{F}=T \ln \left[2 \sinh \left(\frac{\hbar \omega}{2 T}\right)\right]
$$

so that canonical thermodynamic entropy

$$
S=-\frac{\partial \mathcal{F}}{\partial T}
$$

coincides with correlational entropy (73). Thermodynamic energy of the effective oscillator is equal to

$$
\mathcal{U}=\mathcal{F}+T S=\hbar \omega\left(\bar{\nu}+\frac{1}{2}\right)=\frac{\hbar \omega}{2} \operatorname{coth} \frac{\hbar \omega}{2 T}
$$

where the mean number of excited effective quanta is given by the Planck formula,

$$
\bar{\nu}=\frac{1}{\exp (\hbar \omega / T)-1}
$$


Let us consider an arbitrary dynamical variable of the original oscillator described by the operator $\mathcal{O}\left(a^{\dagger}, a\right)$,

$$
\left.\overline{\mathcal{O}}=\int d \lambda \mathcal{P}(\lambda)\langle\lambda| \mathcal{O}\left(a^{\dagger}, a\right)\right)|\lambda\rangle=\int d \lambda \mathcal{P}(\lambda) O(\lambda, \lambda),
$$

where the operators $a^{\dagger}$ and $a$ of original quanta are supposed to be normally ordered with respect to the unperturbed vacuum; in the second equality (93) the properties of the coherent state $|\lambda\rangle$ were used. Since the parameters of the effective oscillator depend on temperature (85) via the factor $\sqrt{\sinh \eta}$, the mean value of any operator (93) contains an additional nontrivial temperature dependence. Indeed, using the relations (58) and (69),

$$
\mathcal{P}(\lambda)=\rho(\lambda, \lambda)=\sum_{\nu=0}^{\infty} \rho_{\nu} \phi_{\nu}(\lambda) \phi_{\nu}(\lambda)
$$

we can express the mean value (93) in terms of the effective oscillator

$$
\begin{gathered}
\bar{O}=\sum_{\nu=0}^{\infty} \rho_{\nu}\left(\nu\left|O\left(\frac{\alpha^{\dagger}+\alpha}{\sqrt{2 \sinh \eta}}, \frac{\alpha^{\dagger}+\alpha}{\sqrt{2 \sinh \eta}}\right)\right| \nu\right)= \\
\operatorname{Tr}\left[\rho O\left(\frac{\alpha^{\dagger}+\alpha}{\sqrt{2 \sinh (\hbar \omega / T)}}, \frac{\alpha^{\dagger}+\alpha}{\sqrt{2 \sinh (\hbar \omega / T)}}\right)\right]
\end{gathered}
$$

with the density operator from eq. (88). For example, the mean number of original quanta is given by

$$
\bar{k}=\sum_{\nu=0}^{\infty} \rho_{\nu}\left(\nu\left|\left(\frac{\alpha^{\dagger}+\alpha}{\sqrt{2 \sinh \eta}}\right)^{2}\right| \nu\right)=\frac{\bar{\nu}+1 / 2}{\sinh \eta}=\frac{\mathcal{U}}{\hbar \omega \sinh \eta}=\frac{1}{4 \sinh ^{2}(\hbar \omega / 2 T)}
$$

in agreement with (77). The distribution of the original quanta differs from the Planck formula (92). At a narrow range of noise, $T \ll \hbar \omega, \Lambda \approx \exp (-\hbar \omega / 2 T)$, which corresponds to the quantum low-temperature limit, we obtain a normal quantum result,

$$
\bar{k}=e^{-\hbar \omega / T}, \quad T \ll \hbar \omega .
$$

A broad noise, $\Lambda \approx T / \hbar \omega \gg 1$, leads to the classical limit of high temperature. In this limit, the number of quanta is increasing with temperature quadratically rather than linearly,

$$
\bar{k}=\left(\frac{T}{\hbar \omega}\right)^{2}, \quad T \gg \hbar \omega .
$$


Let the random field applied to an oscillator with the electric charge $e$ be abruptly removed at the moment $t=0$. The oscillator remains excited and starts to radiate electromagnatic waves losing its energy with the rate

$$
\overline{\dot{E}}=\frac{2 e^{2} x_{0}^{2} \omega^{4}}{3 c^{3}} \bar{k}=\frac{2 e^{2} x_{0}^{2} \omega^{4}}{3 c^{3}} \frac{\bar{\nu}+1 / 2}{\sinh (\hbar \omega / T)}=\frac{2 e^{2} x_{0}^{2} \omega^{4}}{3 c^{3}} \frac{1}{4 \sinh ^{2}(\hbar \omega / 2 T)} .
$$

The emission rate directly measures effective temperature. Having no knowledge about the

noise properties and detecting only the frequency $\omega$ and the intensity $\overline{\dot{E}}$ of radiation, we could assume thermal equilibrium inside the radiating system and assign temperature $T_{0}$ by the direct application of the Planck formula instead of the actual distribution (79). It is easy to see that these temperatures are related through

$$
\sinh ^{2}(\hbar \omega / 2 T)=\frac{1}{2} \sinh \left(\hbar \omega / 2 T_{0}\right) e^{\hbar \omega / 2 T_{0}} .
$$

For the narrow noise, two definitions agree,

$$
T_{0} \approx T, \quad T \ll \hbar \omega
$$

However, under conditions of the broad noise, they lead to very different assignments with $T_{0} \gg T$,

$$
T_{0} \approx \frac{T^{2}}{\hbar \omega}, \quad T \gg \hbar \omega
$$

Two definitions can be discriminated in the case of the spectrum of normal modes with different frequencies by the deviations from the Planck formula at the low-frequency edge, if the noise amplitude $F$ is not frequency dependent.

\section{A MANY-BODY EXAMPLE}

Here we illustrate the concept of correlational entropy by calculating this quantity for a realistic many-fermion model with strong interparticle interaction of a variable random strength. We use for this purpose the version of the nuclear shell model which provides the best available description of spectroscopic data for all $s d$-nuclei [47]. The model hamiltonian 
consists of the one-body part given by a spherical mean field due to the closed shell core of ${ }^{16} \mathrm{O}$ and the semiempirical residual two-body interaction determined by 63 reduced matrix elements $\left\langle\left(j_{1} j_{2}\right) J T\|V\|\left(j_{3} j_{4}\right) J T\right\rangle$ allowed in this truncated Hilbert space by the conservation laws of angular momentum $J$ and isospin $T$. Apart from numerous specific nuclear calculations, this model was recently used [23,26, 7] for studying highly excited states beyond the limits of experimental information. One of the methods utilized in this analysis studies the evolution of observables as a function of the strength of the residual interaction [0, 37, 48].

We take as a generic example a set of states with quantum numbers $J^{\pi} T=0^{+} 0$ in the ${ }^{24} \mathrm{Mg}$ nucleus with 4 protons and 4 neutrons in the $s d$-shell model space. The dimension of this set of states is $N=325$. The unperturbed basis $|k\rangle$ is that of simple independent particle configurations which are projected onto correct values of integrals of motion $J$ and $T$. The residual interaction is split into two parts, diagonal and off-diagonal with respect to the basis $|k\rangle$. The diagonal part is included into unperturbed energies in order to lift the degeneracy of bare configurations. The off-diagonal interaction with the overall factor $\lambda$ in front is our "random noise" with $\lambda=0$ and $\lambda=1$ corresponding to the independent particle case and to the realistic strength, respectively.

The many-body hamiltonian is diagonalized as a function of $\lambda$ which provides us with 325 energy terms $E_{\alpha}(\lambda)$. These terms were shown in Fig. 1 of Ref. 37. Multiple avoided level crossings rapidly transform the eigenstates of the hamiltonian into complicated superpositions of many original configurations. The generic signatures of quantum chaos in level statistics, such as nearest level spacing distribution, spectral rigidity, and level curvature distribution, agree with the GOE predictions already at $\lambda \approx 0.3$. The calculation of information entropy in the original mean field basis shows that the evolution of complexity continues so that at $\lambda=1$ the states in the middle of the spectrum are close to the GOE limit of $I=\ln (0.48 N)$.

Using the known eigenfunctions $|\alpha\rangle$ of the system in the original basis $|k\rangle$, we find the density matrix $\rho_{k k^{\prime}}^{(\alpha)}(\lambda)$ of Eq. (2) and construct the ensemble of such matrices for different values of $\lambda$. The uniform averaging over $\lambda$ in the chaotic interval $0.3 \leq \lambda \leq 1$ gives 
the density matrix $\rho^{(\alpha)}$ of Eq. (5). This matrix can be diagonalized and its eigenvalues (occupation numbers) determine correlational entropy (8).

Fig. 3 shows correlational entropy $S^{\alpha}$ of all 325 states ordered according to increasing energy. Although the fluctuations at this dimension are significant, nevertheless we see the same qualitative pattern as the one obtained with the help of information entropy [23,7]. The degree of mixing measured by correlational entropy evolves along the spectrum revealing the regular increase with excitation energy (in finite Hilbert space the statistical properties of eigenstates are symmetric with respect to the middle of the spectrum; in the thermal description [26] the center corresponds to infinite temperature with positive and negative temperature regions on the left and on the right, respectively). The behavior of information entropy and of single-particle entropy defined by the evolution of the singleparticle occupation numbers is essentially the same. However, the absolute scales are very different. As seen in Fig. 3, the typical values of $\tilde{N}_{\alpha}=\exp \left[S^{(\alpha)}\right]$ for the most complicated states are close to 6 which is much lower than the localization length $\exp \left[I^{(\alpha)}\right]$ found with the use of information entropy. From the perturbative analysis of Sect. 3 we know that the number of effectively occupied eigenstates of the density matrix is related to the order

of perturbation theory. Then $\tilde{N}$ can be interpreted in terms of a number of particle-hole excitons created by the spectral evolution. This point deserves to be studied more in detail.

\section{CONCLUSION}

We have discussed some examples of quantum-mechanical systems under the conditions of stationary external noise which was defined by a distribution function of parameters controlling the interaction hamiltonian. The maximum of quantum information on such systems is provided by the density matrix which was found for various cases, analytically in Sect. 3-5 and numerically in Sect. 6. The perturbative treatment of Sect. 3 illustrates the mechanism of equlibration through the consecutive selection of directions in Hilbert space which form the eigenbasis of the density matrix. In other examples the weakness of the 
interaction was not assumed, and the solution was exact.

We have used correlational (von Neumann) entropy as a tool for measuring the degree of disorder and complexity of stationary states. By definition, this entropy vanishes for pure quantum states. Therefore it characterizes the system in a given noise ensemble when the stationary states are mixed. In general, the arising configuration with certain occupation numbers of the eigenstates of the density operator does not coincide with thermal equilibrium as defined by canonical Gibbs ensembles. Accordingly, correlational entropy is not equal to thermodynamic entropy. Moreover, correlational entropy is calculated for the individual energy terms which evolve adiabatically as a function of the noise strength. The resulting state is in general different for different terms.

It would be interesting to determine the conditions for the external noise which would give the same equilibrated state as in the heat bath. It is assumed usually that the necessary ingredient is the continuous spectrum of the normal modes represented in the spectral expansion of noise [49]. We deal with the stationary noise represented by random parameters in the hamiltonian. In Sect. 5 we have however shown that the ground state of a harmonic oscillator in a random uniform field can be described as an equilibrated thermal state of an effective oscillator with temperature determined by the Gaussian width of the field distribution. The situation here is similar to that in many-body physics where the interacting system can be modeled [50] by a gas of dressed quasiparticles with properties depending on temperature (in our case the energy spectrum of the oscillator is not renormalized but the coordinate scale is determined by noise and changes with effective temperature). The difference between a simply heated oscillator in a thermostat and an oscillator excited by a Gaussian noise and described with the aid of effective temperature might be important for the problems of multiple meson production 46,51] in high energy collisions. We can remind also that the notion of the effective quantum oscillator appears naturally in the problem of a uniformly accelerated observer in the Minkowski world [52,53. An observer falling with the proper acceleration $g$ sees the Minkowski vacuum as a black body emitter with the effective temperature $T=\hbar g /(2 \pi c)$. This consideration is closely related to the Hawking black hole 
radiation [54]. However, in those cases, one has squeezed rather than coherent states of the oscillator (in terms of the unperturbed system they are produced by the source creating the quanta pairwise which is described with the help of the Bogoliubov transformation).

Representing the complexity of individual quantum terms, our invariant correlational entropy can be juxtaposed to representation-dependent information entropy. We drew the attention of the reader to their similarity and distinction in various applications. Although they are formally quite different and may react in a different way on the change of parameters of a simple regular system, Sect. 4, they behave qualitatively similar in a complicated system, Sect. 6, where one sees the standard signatures of quantum chaos. Due to similarity of adjacent states in the chaotic regime [7], both entropies are smooth functions of excitation energy and therefore can be considered as thermodynamic variables. Both entropies carry information on complexity of individual states and its evolution along the spectrum. Quantitatively, information entropy (in the appropriate basis!) expresses this complexity in terms of a number of mixed simple configurations whereas correlational entropy measures essentially similar properties in larger blocks as a number of classes of states effectively mixed by external noise. Of course, one should remember that information entropy refers to a given hamiltonian while correlational entropy describes a "system plus noise" complex. The further studies will bring the more deep insight into the problem.

The authors acknowledge support from the NSF grants 94-03666, 95-12831 and 96-05207. One of us (V.V.S) thanks National Superconducting Cyclotron Laboratory for hospitality; he acknowledges financial support from INTAS, grant 94-2058. V.V.S. and V.Z. thank V.F. Dmitriev and V.B. Telitsin for interesting and useful discussions; they are also grateful to D.V. Savin and V.V. Zelevinsky for assistance. 


\section{REFERENCES}

[1] M. Ohya and D. Petz, Quantum Entropy and Its Use (Springer-Verlag, Berlin - Heidelberg, 1993).

[2] A. Wehrl, Rev. Mod. Phys. 50, 221 (1978).

[3] L.D. Landau and E.M. Lifshitz, Statistical Physics (Pergamon Press, Oxford, 1958).

[4] K. Blum, Density Matrix Theory and Applications (Plenum Press, New York - London, 1981).

[5] L. van Hove, Physica 21, 517 (1955); 23, 441 (1957); 25, 268 (1959).

[6] M. Srednicki, Phys. Rev. E30, 888 (1994).

[7] V. Zelevinsky, B.A. Brown, N. Frazier and M. Horoi, Phys. Rep. 276, 85 (1996).

[8] R.S. Ingarden and K. Urbanik, Acta Phys. Pol. 21, 281 (1962).

[9] M.D. Srinivas, J. Math. Phys. 19, 1952 (1978).

[10] R. Kosloff and S.A. Rice, J. Chem. Phys. 74, 1340 (1981); 86, 2153 (1982).

[11] P. Pechukas, J. Chem. Phys. 86, 2239 (1982).

[12] R. Alicki and M. Fannes, Lett. Math. Phys. 32, 75 (1994).

[13] M.H. Partovi, Phys. Lett. A151, 389 (1990).

[14] W. Słomczyński and K. Życzkowski, J. Math. Phys. 35, 5674 (1994); Erratum 36, 5201 (1995).

[15] A.J. Lichtenberg and M.A. Lieberman, Regular and Stochastic Motion (Springer, New York, 1989).

[16] J.W. Helton and M. Tabor, J. Phys. A18, 2743 (1985).

[17] G. Lindblad, in Quantum Probability and Applications III, Lecture Notes in Math. 1303 
(Springer, Berlin, 1988) p. 183.

[18] C. Beck and D. Graudenz, Phys. Rev. A46, 6265 (1992).

[19] F.J. Yonezawa, Non-Cryst. Solids 35 \& 36, 29 (1980).

[20] J. Reichl, Europhys. Lett. 6, 669 (1988).

[21] F.M. Izrailev, Phys. Rep. 196, 299 (1990).

[22] V.G. Zelevinsky, Nucl. Phys. A555, 109 (1993).

[23] V. Zelevinsky, M. Horoi and B.A. Brown, Phys. Lett. B350, 141 (1995).

[24] I.C. Percival, J. Phys. B6, L229 (1973).

[25] V.Zelevinsky, Annu. Rev. Nucl. Part. Sci. 46, 237 (1996).

[26] M. Horoi, V. Zelevinsky and B.A. Brown, Phys. Rev. Lett. 74, 5194 (1995).

[27] P. Pechukas, Phys. Rev. Lett. 51, 943 (1983).

[28] T. Yukawa, Phys. Lett. 116, 227 (1986).

[29] A. Szafer and B.L. Altshuler, Phys. Rev. Lett. 70, 587 (1993).

[30] B.D. Simons and B.L. Altshuler, Phys. Rev. Lett. 70, 4063 (1993); Phys. Rev. B48, $5422(1993)$.

[31] B.D. Simons, P.A. Lee and B.L. Altshuler, Phys. Rev. Lett. 70, 4122 (1993); 72, 64 (1994).

[32] Y. Alhassid and H. Attias, Phys. Rev. Lett. 74, 4635 (1995).

[33] P. Gaspard et.al., Phys. Rev. A42, 4015 (1990).

[34] J. Zakrzewski and D. Delande, Phys. Rev. E47, 1650 (1993).

[35] F. von Oppen, Phys. Rev. Lett. 73, 798 (1994). 
[36] D. Kusnezov and C.H. Lewenkopf, Phys. Rev. E53, 2283 (1996).

[37] D. Kusnezov, B.A. Brown and V. Zelevinsky. Phys. Lett. B385, 5 (1996).

[38] F.J. Dyson, J. Math. Phys. 3, 1191 (1962).

[39] F. Haake, Quantum Signatures of Chaos (Springer, New York, 1991).

[40] V.V. Sokolov and V.G. Zelevinsky, Nucl. Phys. A304, 562 (1989).

[41] K.R.W. Jones, J. Phys. A23, L1247 (1990).

[42] A. Bulgac, G. Do Dang and D. Kusnezov, Ann. Phys. 242, 1 (1995).

[43] G. Casati et al., Phys. Rev. Lett. 64, 1 (1990).

[44] Y.V. Fyodorov and A.D. Mirlin, Phys. Rev. Lett. 67, 2405 (1991).

[45] J.N. Hollenhorst, Phys. Rev. D19, 1669 (1979).

[46] I.V. Andreev, M. Plumer and R.M. Weiner, Phys. Rev. Lett. 67, 3475 (1991).

[47] B.A. Brown and B.H. Wildenthal, Annu. Rev. Nucl. Part. Sci. 38, 29 (1988).

[48] N. Frazier, B.A. Brown and V. Zelevinsky, Phys. Rev. C54, 1665 (1996).

[49] J.P. Gordon, L.R.Walker, W.H. Louisell, Phys. Rev. 130, 806 (1963).

[50] L.D. Landau, JETP, 11, 592 (1941); 30, 1058 (1956) [English translation Collected Papers by L.D. Landau, ed. D. Ter Haar (Gordon \& Breach, New York, 1965) pp. 301; 723]; P. Noziéres, Theory of Interacting Fermi Systems (Benjamin, New York, 1964).

[51] B.A. Bambah and M.V. Satyanarayana, Phys. Rev. D38, 2302 (1988).

[52] P.C.W. Davies, J. Phys. A8, 609 (1975); Rep. Prog. Phys. 41, 1313 (1978).

[53] R.F. Bishop and A. Vourdas, J. Phys. A19, 2525 (1986).

[54] D.W. Sciama, P. Candelas and D. Deutsch, Adv. Phys. 30, 327 (1981). 


\section{Figure captions}

Figure 1. Correlational entropy of the two-level system (16) as a function of the ratio $\tau=2 \mathrm{~V} / \Lambda$ of the strength of the mixing interaction $2 \mathrm{~V}$ to the range of the random fluctuation $\Lambda$ of the level positions; parts a, $b, c$ and $d$ correspond to the values $\chi=\epsilon / \Lambda=0.2,0.6,1$ and 5 , respectively, where $\epsilon$ is the static level spacing.

Figure 2. Correlational entropy of the two-level system (16) as a function of the random perturbation strength $\chi^{-1}$ for four values of the intrinsic mixing strength $\zeta=\tau / \chi$ : 0.01 (solid line), 0.1 (dots), 2 (dash), 10 (dot-dash). All the curves asymptotically approach the limiting value $\ln 2$; the rate of approach is slower for larger values of the strength parameter.

Figure 3. Correlational entropy of $325 J^{\pi} T=0^{+} 0$ states in the sd-shell model for the ${ }^{24} \mathrm{Mg}$ nucleus. The states are ordered in increasing energy. 


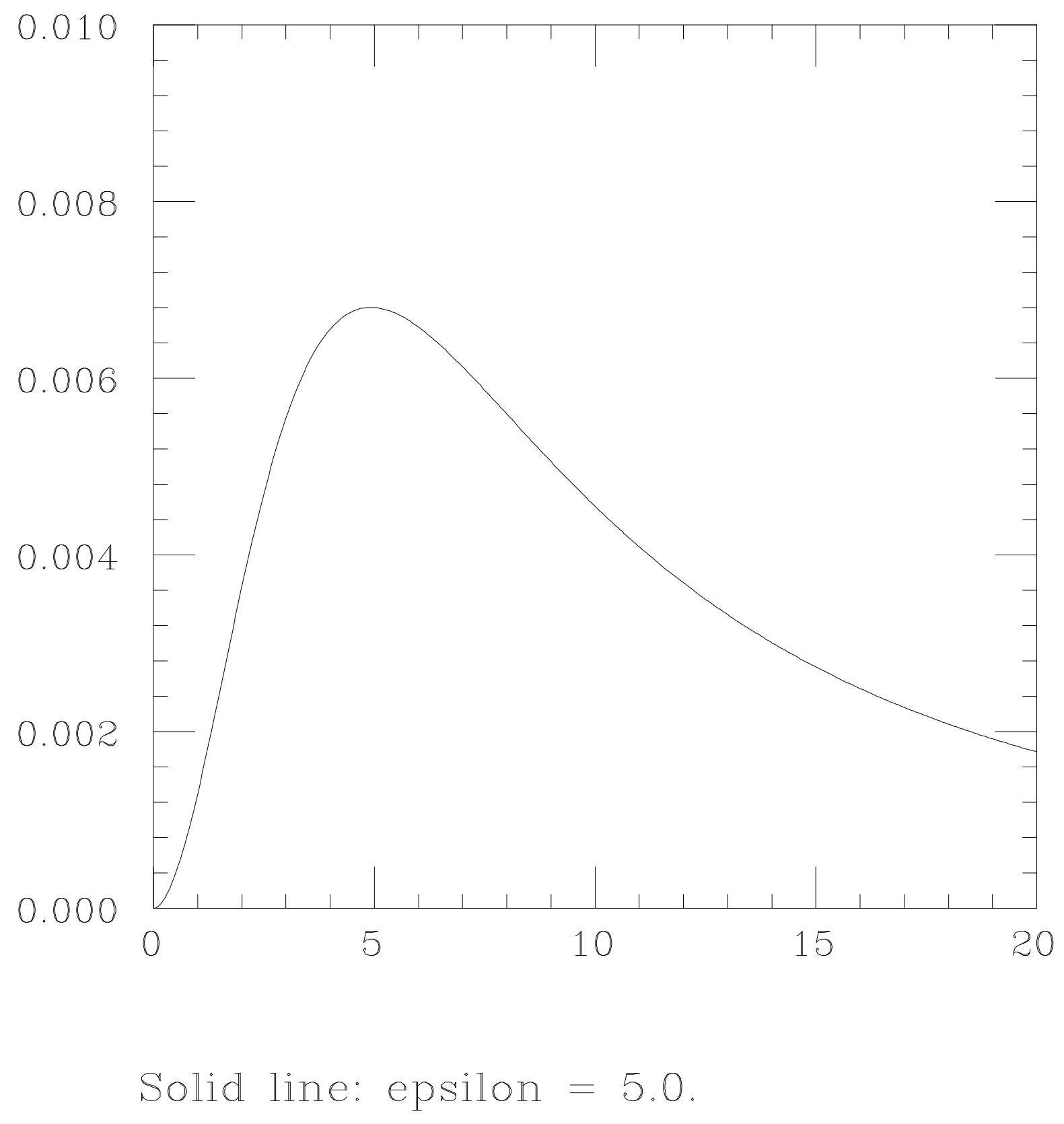




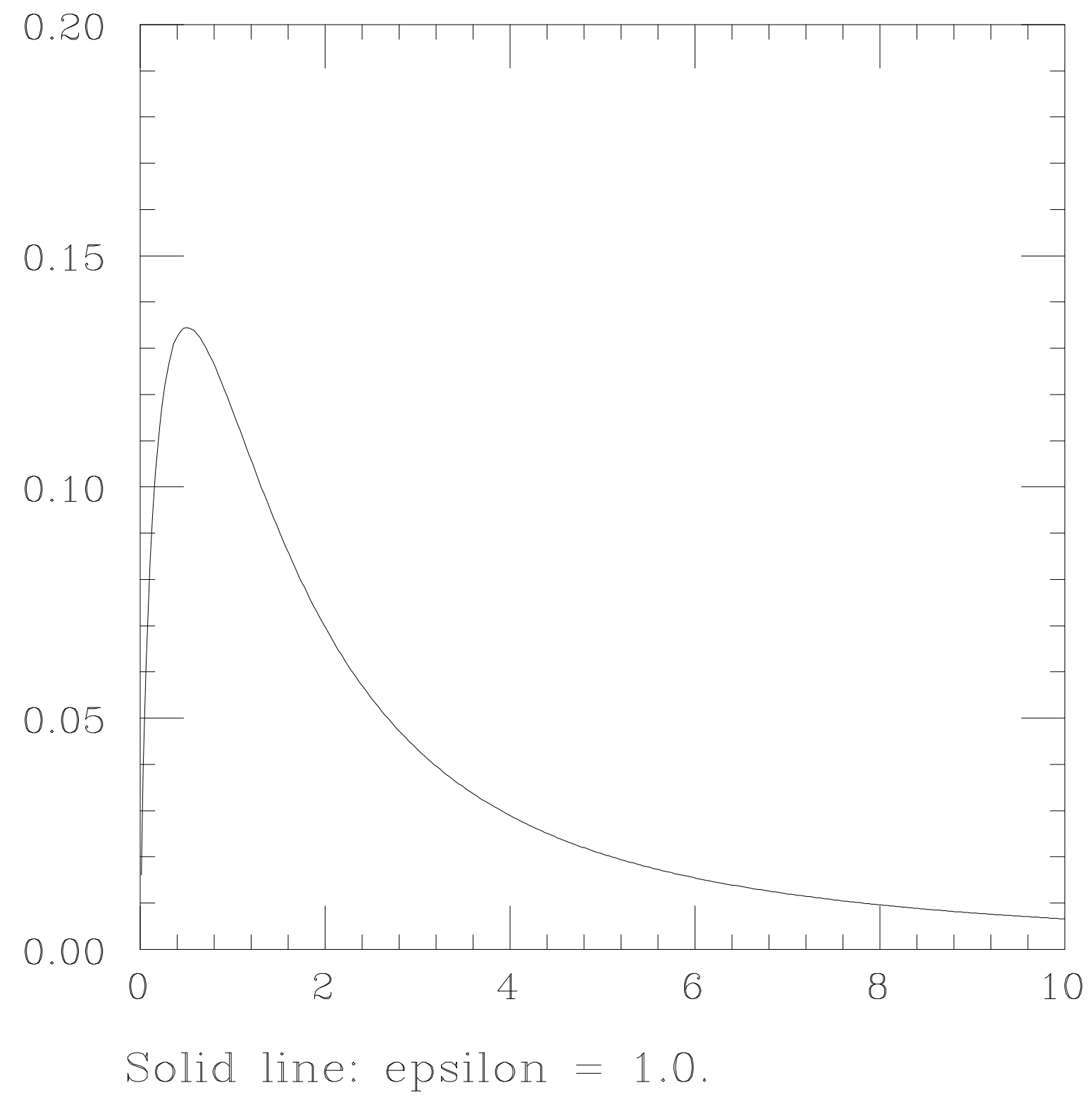




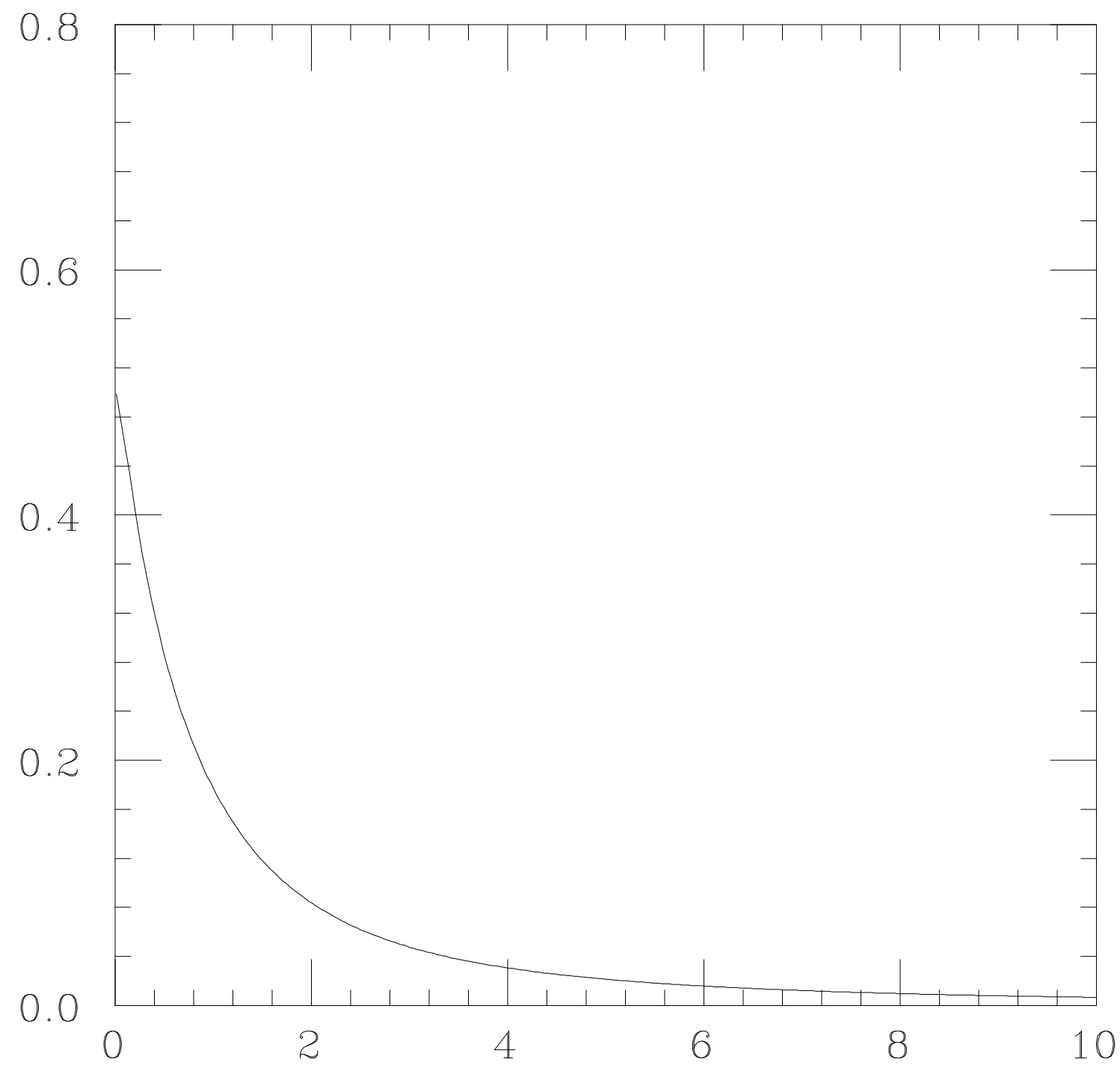

Solid line: epsilon $=0.6$. 


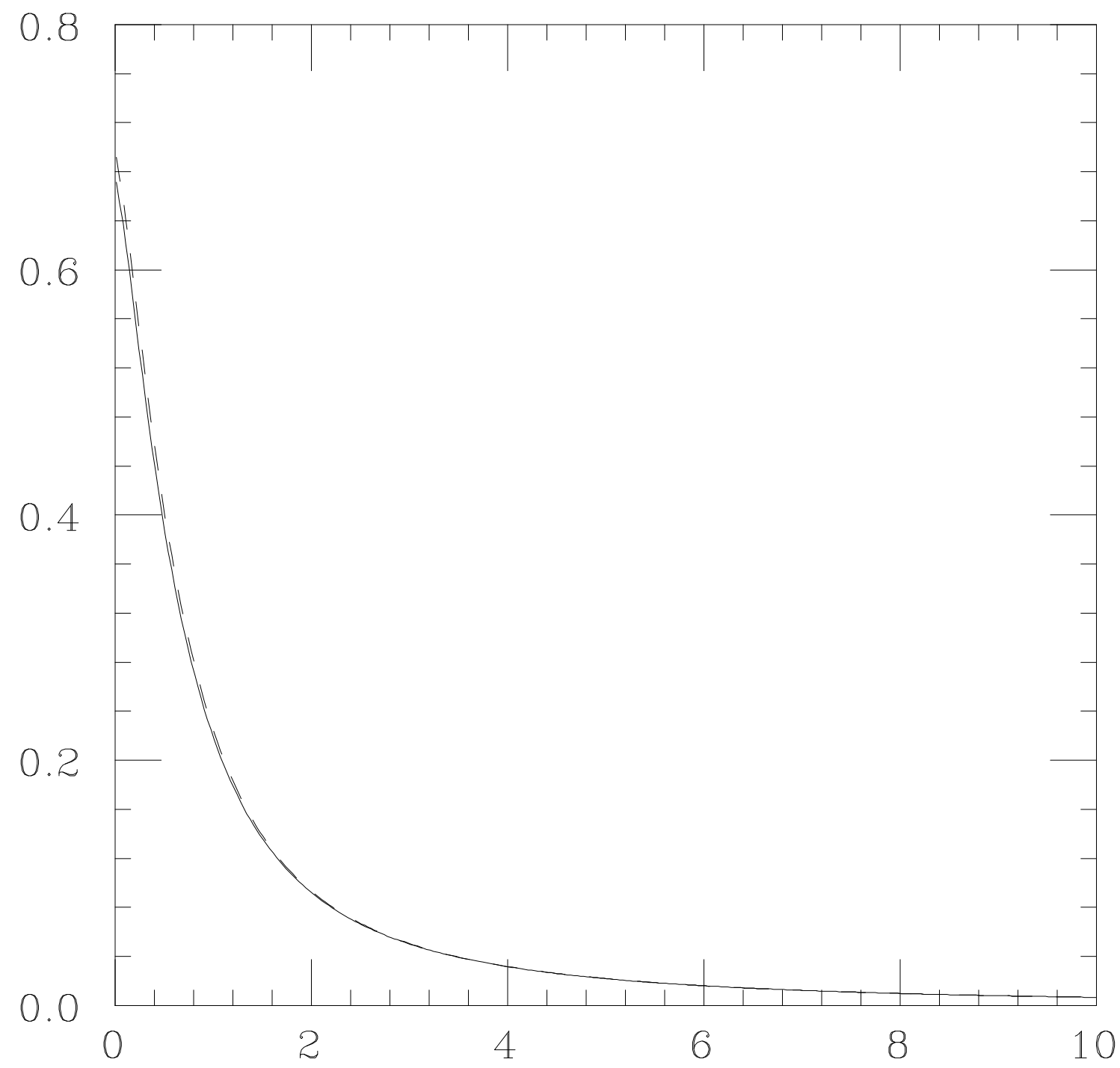

Solid line: epsilon $=0.2$, dashed: 0.0 . 


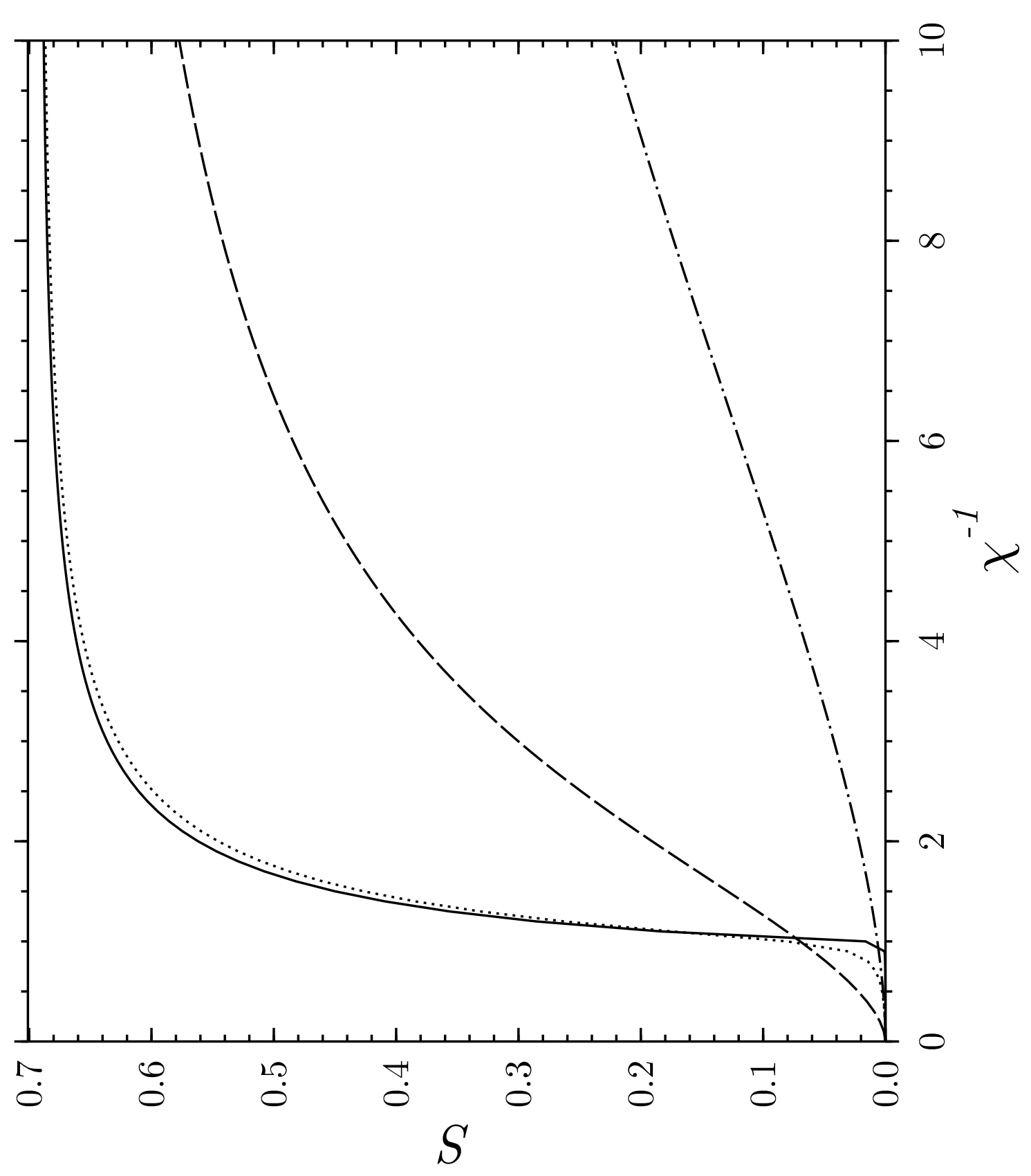

